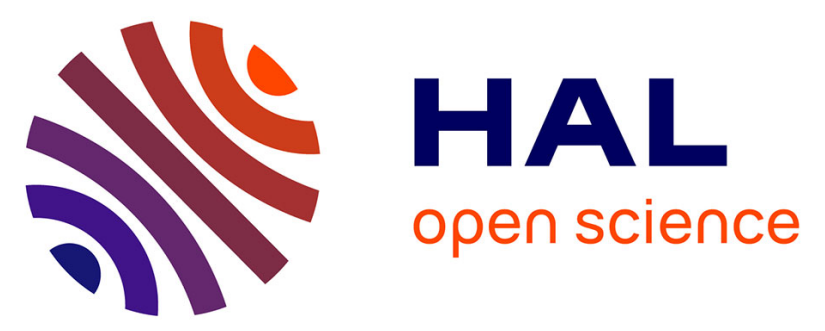

\title{
Magnetic resonance imaging method based on magnetic susceptibility effects to estimate bubble size in alveolar products: application to bread dough during proving.
}

François de Guio, Maja Musse, Hugues Benoit-Cattin, Tiphaine Lucas, Armelle Davenel

\section{To cite this version:}

François de Guio, Maja Musse, Hugues Benoit-Cattin, Tiphaine Lucas, Armelle Davenel. Magnetic resonance imaging method based on magnetic susceptibility effects to estimate bubble size in alveolar products: application to bread dough during proving.. Magnetic Resonance Imaging, 2009, 27 (4), pp.577-85. 10.1016/j.mri.2008.08.009 . hal-00454523

\section{HAL Id: hal-00454523 \\ https://hal.science/hal-00454523}

Submitted on 8 Feb 2010

HAL is a multi-disciplinary open access archive for the deposit and dissemination of scientific research documents, whether they are published or not. The documents may come from teaching and research institutions in France or abroad, or from public or private research centers.
L'archive ouverte pluridisciplinaire HAL, est destinée au dépôt et à la diffusion de documents scientifiques de niveau recherche, publiés ou non, émanant des établissements d'enseignement et de recherche français ou étrangers, des laboratoires publics ou privés. 
1 Magnetic resonance imaging method based on magnetic

2 susceptibility effects to estimate bubble size in alveolar products:

3 Application to bread dough during proving

4

5 François De Guio a,b,*

6 Maja Musse ${ }^{\mathrm{a}, \mathrm{b}}$

7 Hugues Benoit-Cattin ${ }^{\mathrm{c}}$

8 Tiphaine Lucas ${ }^{\mathrm{a}, \mathrm{b}}$

9 Armel Davenel $^{\mathrm{a}, \mathrm{b}}$

$10 \quad{ }^{a}$ Cemagref, UR TERE, 17 avenue de Cucillé, 35044 Rennes, France

$11{ }^{\mathrm{b}}$ Université européenne de Bretagne, France

$12{ }^{\mathrm{c}}$ CREATIS, UMR CNRS 5220, Inserm U630, Université Claude Bernard Lyon 1, INSA Lyon,

13 Bât. Blaise Pascal, 69621 Villeurbanne, France

14

$15 *$ Corresponding author. E-mail address: francois.de-guio@cemagref.fr

16

17 


\section{Abstract}

Magnetic resonance imaging has proven its potential application in bread dough and

20 gas cell monitoring studies, and dynamic processes such as dough proving and baking can be 21 monitored. However, undesirable magnetic susceptibility effects often affect quantification 22 studies, especially at high fields. A new low field method is presented based on local 23 assessment of porosity in spin-echo imaging, local characterization of signal loss in gradient24 echo imaging and prediction of relaxation times by simulation to estimate bubble radii in 25 bread dough during proving. Maps of radii showed different regions of dough constituting 26 networks which evolved during proving. Mean radius and bubble distribution were assessed 27 during proving.

29 Key Words: magnetic susceptibility; MRI simulation; field inhomogeneity; gas bubble; gas 30 cell; bread; dough; proving; fermentation 


\section{Introduction}

Magnetic susceptibility effects are of increasing interest in Magnetic Resonance Imaging (MRI). Traditionally, as field inhomogeneities induce geometry and intensity distortions in spin-echo (SE) imaging and signal loss in gradient-echo (GE) imaging [1, 2], susceptibility artefacts have been considered undesirable and therefore subjected to correction [3]. However, there are number of emerging techniques and applications using magnetic susceptibility effects and $\mathrm{T}_{2}{ }^{*}$-weighted imaging. The difference in magnetic susceptibility between deoxygenated and oxygenated blood, termed the BOLD effect, led to functional MRI which is widely used in cognitive neuroscience [4]. Susceptibility Weighted Imaging (SWI) uses the original phase image to enhance contrast between tissues with different susceptibilities [5]. This technique has been applied to studies of brain tumors, trauma, vascular malformations and for quantification of brain iron [6]. Superparamagnetic iron oxide (SPIO) particles create intense magnetic field distortions within and around cells, leading to irreversible signal dephasing in GE sequences. SPIO particles have been exploited as contrast agents for non-invasive cell tracking to determine their biodistribution in different organs [710]. Susceptibility studies have been applied to material characterization $[11,12]$ and particle identification in industrial systems [13].

The potential of gas microbubbles as an in vivo intravascular susceptibility contrast agent for MRI has been demonstrated [14]. Moreover, differences in magnetic susceptibility $(\Delta \chi)$ between gas bubbles and a water environment create field inhomogeneities which induce intravoxel dephasing and associated signal loss characterized by a $\mathrm{T}_{2} *$ shortening in GE imaging. However, the presence of air bubbles in several food products has not to date been the focus of studies of susceptibility effects. Understanding bubble mechanics is very important with a wide range of foamed food products. Bubble studies make possible the optimization of process design, contribute to the development of strategies for deaeration and 
are important in the area of texture and sensory analysis [15]. It can be anticipated that air or gas bubbles would be natural markers or contrast agents. Moreover, bubbles are part of a product and thus could be considered as a signature of the medium.

Proving flour dough is an essential stage in breadmaking. As the final character of most bakery products depends on the creation and control of gas bubble structures in the unbaked matrix, improving the understanding of the nucleation and growth of bubbles is of major interest [16]. MRI is a particularly suitable tool to study bread dough as it is non-invasive (essential for dough monitoring during proving as the alveolar structure is fragile and may collapse if intrusive measurement techniques are used), and provides a good trade-off between spatial and time resolution [17-20]. Dough samples can thus be quite large and imaged with high resolution during proving [20]. Even the entire baking process can be monitored with MRI [21]. Bonny et al. [17] used high-field (9.4 T) magnetic resonance microscopy along with routine mathematical morphology to characterize the proving process noninvasively. They faced critical susceptibility effects responsible for severe geometrical distortions due to the heterogeneous structure characterized by many interfaces. Different stages of fermentation could be identified, with a general increase in bubbles, but no quantitative assessment of bubble size could be made. Van Duynhoven et al. [20] illustrated the ability of MRI and image analysis to assess gas cell development in the growth of the dough during proving. Basically, the size of each cell was determined by counting the number of pixels, thus presupposing high resolution and hence small fields of view. Moreover, this method does not account for susceptibility effects that are especially disrupting at $4.7 \mathrm{~T}$ and are responsible for geometry and intensity distortions in SE imaging. X-ray tomography has also proved its ability in analyzing bubble growth during bread making [22]. However, this technique is only sensitive to density, and imaged samples have to be small $(<10 \mathrm{~mm})$. 
The aim of the study reported here was to characterize the alveolar structure of bread dough and its evolution during proving using susceptibility effects. Grenier et al. [18] assessed local dough porosity (volume of gas per volume of dough) during proving. However, nothing can be inferred about bubble size for a given value of porosity, as different cell distributions can be obtained [23, 24]. We therefore developed an original method to estimate bubble size based on the local porosity assessed in SE imaging and on the susceptibility effects in GE imaging. MRI simulation was used to predict signal loss for virtual networks of gas bubbles embedded in a water matrix. A low magnetic field $\left(B_{0}=0.2 \mathrm{~T}\right)$ was used in order to avoid the severe geometric distortions encountered at high field in SE imaging and critical signal losses in GE imaging as the heterogeneous structures were characterized by many susceptibility interfaces [17]. The dynamic side of bread proving requires acquisition to be quite fast, leading to a limited number of acquisitions, limited resolution and an appropriate hypothesis to compute maps of interest.

Section 2 reports how relaxation times affected by susceptibility were predicted with simulation. Section 3 describes the materials and methods used with bread dough preparations, the MRI protocol, computation of the maps and the principle of the estimation algorithm. Section 4 presents the results on non-yeasted bread dough and yeasted dough during proving, followed by a discussion in section 5 .

\section{Prediction of relaxation times by simulation}

The algorithm developed for estimation of bubble size was partly based on simulation

100 results. This section introduces theoretical considerations and describes the simulation

101 protocol. We demonstrate how relaxation times can be modeled at $0.2 \mathrm{~T}$ and what can happen at $1.5 \mathrm{~T}$. 


\subsection{Theoretical considerations}

104 We first verified that the static dephasing regime conditions for spherical magnetic 105 perturbers of radius $r$ as described by Yung et al. [25] were largely met, as $1 / \tau<<\delta \omega$ with $\tau=$

$106 r^{2} / D$ and $\delta \omega=\gamma \Delta \chi B_{0} / 3, D$ being the translational diffusion coefficient of spins taken as the water diffusion coefficient and $\gamma$ the proton gyromagnetic ratio. In fact, $D$ is even smaller in 108 bread dough.

Microscopic field inhomogeneities (i.e. magnetic field inhomogeneities over distances

110 with orders of magnitude smaller than the voxel size) are responsible for irreversible signal 111 decay $\left(R_{2}=1 / T_{2}\right)$ and mesoscopic field inhomogeneities (from perturbers smaller than the 112 voxel size but greater than the diffusion length) contribute to $R_{2}{ }^{\#}$, the reversible portion of $R_{2}{ }^{*}$ $113\left(R_{2}{ }^{*}=R_{2}+R_{2}{ }^{\#}=1 / T_{2}{ }^{*}\right)[3,26]$. Macroscopic field inhomogeneities are not present in $T_{2}{ }^{*}$

114 definition and induce non-exponential signal decay $[3,27]$. Macroscopic field 115 inhomogeneities can typically arise from air inclusions or ferromagnetic objects. Finally, 116 assuming a constant proton density across the voxel $(\rho(\boldsymbol{r})=\rho)$, signal decay in a voxel of 117 volume $V$ is expressed in Eq. (1):

$$
S(t)_{\text {voxel }}=\rho / V \cdot e^{-R_{2}^{*} t} \cdot \sin c\left(\gamma g_{x} l_{x} / 2\right) \cdot \sin c\left(\gamma g_{y} l_{y} / 2\right) \cdot \sin c\left(\gamma g_{z} l_{z} / 2\right)
$$

119 where $l_{x}, l_{y}, l_{z}$ are the voxel dimension, and field inhomogeneities along one direction $i$ are 120 expressed with a linear gradient $\left(\Delta B_{i}=g_{i} . i\right)$. In this study, we defined $T_{2} \chi$ as the constant of 121 the exponential curve fitting the initial part of the susceptibility-induced signal decay.

122 Microscopic, mesoscopic and macroscopic inhomogeneities were thus taken into account in 123 the approximation of $T_{2} \chi$.

\subsection{Simulation protocol} The purpose of the simulation was to investigate quantitatively the signal loss in GE

126 MR images induced by networks of gas bubbles embedded in water. We used the SIMRI MRI 
127 simulator as described by Benoit-Cattin et al [28]. In a previous study [27], intravoxel

128 modeling and associated signal decay were quantitatively assessed and experimentally

129 validated in the case of a single well-resolved air-filled cylinder and in the case of a network

130 of small interacting air-filled cylinders. An overview of the simulation framework is 131 presented in Fig. 1. A 3D virtual object was defined by radius $(r)$ and center to center distance 132 between bubbles $(c c d)$ expressed in pix $_{\text {obj }}$ (i.e. the pixel in the $256^{3}$ object space), difference in 133 magnetic susceptibility $(\Delta \chi)$ and number of bubbles in each dimension $(N b)$. The additive 134 property of the magnetic field, and the analytical formulation of field inhomogeneities created 135 by one sphere [1], were combined to compute the relative field inhomogeneities $\left(\Delta B / B_{0}\right)$. 136 From this map, SIMRI provides simulated MR images with susceptibility effects. At this 137 stage, object parameters such as spin-lattice relaxation time $T_{1}$, spin-spin relaxation time $T_{2}$, 138 and proton density $\rho$ could be defined. NMR experiments (Minispec PC 120, Bruker SA, 139 Wissembourg, France) on the bread dough showed several $T_{1}$ and $T_{2}$ components leading to 140 multi-exponential decay, as previously described [29, 30]. The first components were smaller 141 than the echo times (TE) used in this study. As a single value for relaxation times is necessary 142 in simulation, weighted averaging of the relaxation times of the different components was 143 performed, resulting in $T_{1}=100 \mathrm{~ms}$ and $T_{2}=20 \mathrm{~ms}$. Lodi et al. [31] assessed $T_{2}$ values from 144 spin-echo images by fitting time-series points pixel-by-pixel, resulting in $T_{2}$ maps of soy 145 bread (mean values around $18 \mathrm{~ms}$ ) consistent with this averaging. Simulation parameters such 146 as main magnetic field $\left(B_{0}=0.2 \mathrm{~T}\right)$, pixel bandwidth $(B W=279 \mathrm{~Hz} /$ pixel $)$ and repetition time $147(T R=300 \mathrm{~ms})$ were set to match real experimental conditions. By defining the number of 148 pixels constituting the simulated image, we could define the relative proportion of the bubble 149 radius in the simulated image pixel ( pix $_{\text {ima }}$ ). For instance, considering the high-resolution 150 object used as an example in Fig. $1\left(r=4\right.$ pix $_{\mathrm{obj}}, c c d=12$ pix $_{\mathrm{obj}}, \Delta \chi=-9.05 \mathrm{ppm}, \mathrm{Nb}=17$ in 151 the $256^{3}$ object space), a $32 \times 32$ slice simulated image resulted in $r=0.5$ pix ima,$c c d=1.5$ 
$152 \operatorname{pix}_{\text {ima }}\left(r=0.125\right.$ pix $_{\text {ima }}, c c d=0.375$ pix $_{\text {ima }}$ with a $8 \times 8$ simulated image $)$. For each object, GE 153 images were simulated at different increasing $T E$ from 4 to $12 \mathrm{~ms}$. The mean gray level was 154 then computed on regions of interest (ROI) enclosing several bubbles (visible on Fig. 1). This 155 centred ROI made it possible to avoid boundary effects and to account for the interactions 156 between several bubbles. Good representation of the medium was thus assured with such an

157 ROI as bubbles were regularly spaced. $T_{2} \chi$ was calculated as the constant of the exponential 158 curve fitting the initial part of the mean gray level decay computed in the ROI. In fact, objects

159 with large $c c d$ gave quasi-exponential decay even for longer $T E$ (12 ms). As $c c d$ decreased,

160 signal decay was hardly exponential because of high gradients $g_{i}$ in Eq. (1) and $T_{2} \chi$ was 161 assessed on the very first $T E$ values (between 4 and $8 \mathrm{~ms}$ ).

\subsection{Evolution of $T_{2} \chi$ as a function of bubble radius}

163 Our aim was to link the object configuration with $T_{2 \chi} \chi$ values so as to characterize the 164 medium. The initial question was to find out whether small or large intravoxel bubbles 165 engendered a similar signal loss for the same porosity (i.e. the gas volume fraction in the 166 voxel). We therefore gathered all $T_{2} \chi$ values relative to the different object configurations as a

167 function of bubble radius at the different porosities shown in Fig. 2.a. The same curve shape 168 was observed for each porosity. The first plateau indicated that a similar initial signal loss was 169 measured for small radii, i.e. $T_{2} \chi$ was only dependent on porosity. Then, a rapid increase in $170 T_{2} \chi$ leveled off on a second plateau; note that the lower the porosity the smaller the radii 171 corresponding to $T_{2} \chi$ values as they started to increase. This was appropriate to our study as 172 the initial mean porosity of bread dough was around $10 \%$ and overall porosity and bubbles 173 increased during proving. One significant feature was that $T_{2} \chi$ was sensitive to porosity and 174 intravoxel structure according to bubble size. 

value; a typical sigmoid function being defined in Eq. (2).

$$
f(x)=\frac{1}{1+e^{-\lambda x}}
$$

As curves were not centered but shifted along the radius axis, we defined $r_{\text {break }}$ as the radius corresponding to the symmetrical point of the curve. As the amplitude between low and high radius values was not $1, K$ was introduced as multiplying factor. Finally, $T_{2} \chi_{\text {init }}$ was defined as $T_{2} \chi$ offset, i.e. the value of $T_{2} \chi$ for the smallest radius. Using these new variables and Eq. (2), $T_{2} \chi$ as a function of radius is expressed in Eq. (3):

$$
T_{2 \chi}(r)=T_{2 \chi \text { init }}+K \cdot \frac{1}{1+e^{-\lambda\left(r-r_{\text {break }}\right)}}
$$
to best fit the data according to the least mean square criterion (using Marquardt's algorithm in TableCurve2D® software, Jandel Scientific, version 5). Each parameter separately was

188 then studied as a function of porosity. $T_{2} \chi_{\text {init }}$ and $K$ (Fig. 3.a), $r_{\text {break }}$ and $\lambda$ (Fig. 3.b) were

189 drawn up and found to be closely related to porosity with second order polynomial functions $\left(\mathrm{R}^{2}\left(T_{2} \chi_{\text {init }}\right)=0.995, \mathrm{R}^{2}(K)=0.988, \mathrm{R}^{2}\left(r_{\text {break }}\right)=0.994\right.$ and $\left.\mathrm{R}^{2}(\lambda)=0.993\right)$.

$$
T_{2 \chi}(r, \varepsilon)=T_{2 \text { xinit }}(\varepsilon)+K(\varepsilon) \cdot \frac{1}{1+e^{-\lambda(\varepsilon)\left(r-r_{\text {break }}(\varepsilon)\right)}}
$$

With

$$
T_{2 \text { xinit }}(\varepsilon)=27.1 \varepsilon^{2}-37.1 \varepsilon+17.2
$$

$$
K(\varepsilon)=-52.9 \varepsilon^{2}+49.3 \varepsilon-4.2
$$

$$
\lambda(\varepsilon)=133.9 \varepsilon^{2}-127.5 \varepsilon+63.6
$$

$$
r_{\text {break }}(\varepsilon)=-1.1 \varepsilon^{2}+1.9 \varepsilon+0.2
$$


Note that the polynomial fitting on $K$ as a function of $\varepsilon$ was accurate and determined for

196 porosity values greater than $10 \%$. It was obvious that a homogeneous object without bubbles

$197(\varepsilon=0)$ would normally result in a zero multiplying factor $(K)$. The same was valid for $r_{b r e a k}$,

198 whose limit for low porosities should be zero. Moreover, zero porosity in the $T_{2} \chi_{\text {init }}$ expression

199 corresponded to the $T_{2} *$ value, i.e. the exponential signal decay constant representing the

200 signal loss due to the mesoscopic field inhomogeneities.

\subsection{Signal losses at $1.5 T$}

Although a low magnetic field was used, we felt that it could be of interest to predict signal losses in simulation at a higher field. The same simulations were therefore undertaken

204 at $1.5 \mathrm{~T}$ and the results are summarized in Fig. 2.b. Susceptibility was obviously stronger as 205 field inhomogeneities were proportional to the main magnetic field. When porosity was equal, $206 T_{2} \chi_{\text {init }}$ times were considerably shortened compared to the situation at $B_{0}=0.2 \mathrm{~T}$. Indeed, if $\varepsilon$ $207=0.12$, simulations gave the followings: $T_{2} \chi_{\text {init }}=12.65 \mathrm{~ms}$ at $B_{0}=0.2 \mathrm{~T}$ and $T_{2} \chi_{\text {init }}=3.8 \mathrm{~ms}$ at $208 B_{0}=1.5 \mathrm{~T}$. The value of $B_{0}$ did not seem to impact on $r_{b r e a k}$ value. However, the $K$ factor was 209 significantly increased ( 1.35 at $B_{0}=0.2 \mathrm{~T}$ against 5.11 at $B_{0}=1.5 \mathrm{~T}$ if $\left.\varepsilon=0.12\right)$. Signal decay 210 was dramatically decreased for higher porosities $(\varepsilon>0.2)$, preventing GE studies at high 211 fields.

We have shown in this section that $T_{2} \chi$ relaxation time could be modeled with a 214 sigmoid function dependent on porosity and radius of bubbles. We therefore used Eqs. $(4,5)$ in the next section to build an estimation algorithm to assess bubble size in bread dough. 


\section{Materials and methods}

\subsection{Sample preparation and experimental procedure}

Bread dough samples were obtained by mixing $2000 \mathrm{~g}$ of wheat flour (Type 55, Moulins Soufflet Pantin), $1140 \mathrm{~g}$ of water, $40 \mathrm{~g}$ of salt, $20 \mathrm{~g}$ of improver and $100 \mathrm{~g}$ of yeast (optional) in a Moretti Forni Grain Spiry 8 dough mixer for 17 min at 100 rotations per minute. The advantages of using non-yeasted dough were to provide a stable object with regard to the acquisition time, with possible comparison of structure with yeasted dough at the initial time of proving. A fraction of gas (mainly air) is incorporated at the mixing stage and porosity at the end of mixing was estimated at $10 \pm 2 \%$ depending on the mixer used for a given recipe [32], with a mean gas bubble size of approximately $0.05 \mathrm{~mm}$ to $0.3 \mathrm{~mm}$ [22].

Dough temperature and water content were checked for the evaluation of the reproducibility between batches as both are known to affect the relaxation signal of dough $[29,30]$. The temperature was $24.5 \pm 0.5^{\circ} \mathrm{C}$, and water content was $45 \pm 3 \mathrm{~g}$ of water per 100 $\mathrm{g}$ of dough. Cylindrical flasks ( $\varnothing=50 \mathrm{~mm}, 70 \mathrm{~mm}$ height $)$ were filled with $50 \mathrm{~g}$ of yeasted dough or $100 \mathrm{~g}$ of non-yeasted dough. A lid was placed over the flask to limit dehydration during measurement. Flasks were then placed in a tunnel within the magnet, equipped with thermal regulation set at $24.5^{\circ} \mathrm{C}$. Internal tunnel temperature was monitored with thermocouples. MRI acquisitions were begun at approximately $\mathrm{t}=7 \mathrm{~min}, \mathrm{t}=0$ referring to the end of mixing.

Cylindrical flasks were also filled with $\mathrm{MnCl}_{2}$ solution, the concentration $(1287.2 \mu \mathrm{M})$ being adjusted to obtain a $T_{2}$ value close to that of bread dough, i.e. $T_{2}=20 \mathrm{~ms}$. SE images of the phantom were used to normalize dough MR images to correct for inherent magnet and coil spatial inhomogeneities. 


\subsection{MRI device and parameters}

A $0.2 \mathrm{~T}$ electromagnet scanner in open configuration (Magnetom Open, Siemens, 241 Erlangen, Germany) equipped with a head coil was used to image the bread dough. Double 242 GE sequences $\left(\mathrm{TE}_{1}=4 \mathrm{~ms}, \mathrm{TE}_{2}=12 \mathrm{~ms}\right)$ were performed to assess signal loss and $T_{2} \chi$ during 243 dough proving. SE sequences (TE $=8 \mathrm{~ms}$ ) were used to obtain local porosity. For all 244 sequences, slice thickness $(S T)$, bandwidth $(B W)$, field of view $(F O V)$, matrix size $(N)$, 245 number of accumulations $(A c c)$ and repetition time $(T R)$ were set as follows: $S T=5 \mathrm{~mm}, B W$ $246=279 \mathrm{~Hz} /$ pixel, $F O V=128 \times 128 \mathrm{~mm}^{2}, N=128 \times 128, A c c=4, T R=300 \mathrm{~ms}$. Each sequence 247 lasted $2 \min 33 \mathrm{~s}$, and GE and SE sequences were alternated.

\subsection{Porosity map}

Assuming an initial uniform porosity at a reference time, the porosity of a voxel $(i, j)$ 250 can be estimated from its gray level during proving in SE imaging

$251 \quad[18]: \varepsilon(i, j)=1-\left(1-\varepsilon_{\text {init }}\right) \cdot \frac{S E(i, j)}{M G L_{\text {init }}}$

252 with $\varepsilon_{\text {init }}$ the initial overall porosity $\left(\varepsilon_{\text {init }}=10 \%\right)$ at $t_{\text {init }}=9.5 \mathrm{~min}$, i.e. the time to obtain the 253 first SE image, $M G L_{\text {init }}$ the corresponding mean gray level computed on a reference ROI, and $254 S E(i, j)$ the gray level in the SE image at the location $(i, j)$. We verified that the mean value 255 computed in the porosity map was concordant with the overall porosity computed from the 256 total dough volume measurement.

\section{4. $T_{2 \chi}$ map}

With $G E_{1}(i, j)$ and $G E_{2}(i, j)$ as the GE images at $T E_{1}$ and $T E_{2}$, the $T_{2} \chi$ map can be defined 259 as follows:

260

$$
T_{2 \chi}(i, j)=\frac{\left(T E_{2}-T E_{1}\right)}{\ln \left(\frac{G E_{1}(i, j)}{G E_{2}(i, j)}\right)}
$$


261 In fact, the exponential signal decay constant was estimated from two points only. Prior 262 simulations showed that $T E_{1}=4 \mathrm{~ms}$ and $T E_{2}=12 \mathrm{~ms}$ were suitable for a $0.2 \mathrm{~T}$ magnetic field.

\subsection{Bubble size estimation algorithm}

The algorithm principle was to combine $T_{2} \chi$ and porosity maps to estimate local bubble radii. Indeed, from Eq. (4) we can extract bubble radii $(r)$ and compute the map of radii $r(i, j)$ according to Eq. (8):

$$
r(i, j)=r_{\text {break }}(i, j)-\frac{1}{\lambda(i, j)} \cdot \ln \left(\frac{K(i, j)}{T_{2 \chi}(i, j)-T_{2 \chi_{\text {init }}}(i, j)}-1\right)
$$

$r_{b r e a k}, K, \lambda$ and $T_{2} \chi_{\text {init }}$ were determined for each pixel from the porosity map using Eq. (5).

$T_{2} \chi$ was simulated from a set of several bubbles, i.e. signal loss was characterized in the center of the image to take into account the influence of the neighboring bubbles. This local approach based on several voxels should result in a coherent estimation algorithm, and therefore $r(i, j)$ was not directly computed pixel-by-pixel but through a $3 \times 3$ averaging mask. This allowed replacement of the pixel value by a local average around the pixel of interest. Indeed, the non-linear property of the logarithm function made the subsequent use of an averaging on the map of radii impossible. Prior to computation of Eq. (8), we therefore applied the mask on $r_{b r e a k}, K, \lambda, T_{2} \chi$ and $T_{2} \chi_{\text {init }}$ maps.

\section{Results}

\subsection{Non- yeasted dough}

The porosity map of the non-yeasted dough was computed according to Eq. (6) and is represented in Fig. 4. The largest bubbles were easily detectable and the rest of the dough was quite uniform and dense. Mean porosity on a large reference ROI was found to be $12.6 \%$. The corresponding mean value in the $T_{2 \chi} \chi$ map computed on the same ROI was $12.9 \mathrm{~ms}$. Compared 
284 with simulation results $\left(T_{2} \chi=13 \mathrm{~ms}\right.$ and $\varepsilon=12.5 \%$ in Fig. 2.a), these two values were in 285 good agreement. The $T_{2}$ value of bread dough used for simulation and the general hypothesis 286 of regularly spaced bubbles were justified to reach a good order of magnitude. The bubble 287 size estimation algorithm was then applied to obtain the map of radii depicted in Fig. 5. The 288 large bubbles were even more clearly revealed due to increased contrast compared to the 289 neighboring environment. Fig.5 also demonstrates localized regions of smaller bubbles which 290 were hardly visible in the porosity map (Fig. 4). The map of radii depicted different regions 291 corresponding to different bubble sizes. Smaller bubbles can be seen surrounded by larger bubbles, themselves surrounded by larger bubbles and so on. This radius evolution was spatially reproduced on the dough area, leading to different visible networks. The histogram 294 representing the number of pixels according to their estimated radii extracted from the 295 reference ROI is presented in Fig. 6. A Gaussian probability density function was found to fit 296 well the experimental distribution of bubble size, as shown in Fig. $6\left(\sigma=0.062, \mu=0.37, R^{2}=\right.$ 0.991 with TableCurve2D® software, Jandel Scientific, version 5). The mean value computed

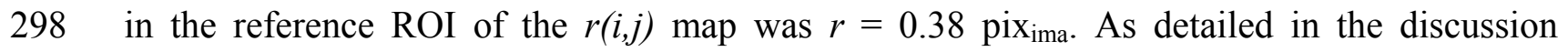
299 section, estimated radius values were subjected to certain limits inherent to the method and 300 values were not systematically significant. However, they can provide relative information 301 about spatial differences (for a given protocol) and between different protocols of dough 302 production.

\subsection{Dough during proving}

As in the previous experiment with the non-yeasted dough, $r(i, j)$ was then computed for dough during proving. The three first maps at $t_{1}=7 \mathrm{~min}, \mathrm{t}_{2}=12.5 \mathrm{~min}$ and $\mathrm{t}_{3}=18 \mathrm{~min}$ after completing mixing are represented in Fig. 7 on the same scale as Fig. 5. Histograms were 307 computed at $t_{1}, t_{2}$ and $t_{3}$, taking the same reference ROI occupying almost all the dough at $t_{1}$ 308 (Fig. 8). As in the non-yeasted dough, distributions were Gaussian and shifted to the higher 
309 radius during proving, consistent with the growth of bubbles under 310 desolubilization/vaporization of $\mathrm{CO}_{2}$, ethanol and water vapor. The average radius estimated

311 from the algorithm as a function of the proving time is also presented in Fig. 9. Bubble size 312 distribution was slightly shifted compared to non-yeasted dough, which may be attributed to 313 the start of fermentation between the end of mixing and the first MRI acquisition (see 314 Materials and Methods section). At $t_{2}$, modification in the dough structure was clearly 315 observed (Fig. 7.b). Groups of bubbles were growing and were identifiable from $t_{1}$ to $t_{3}$ and 316 also for longer proving times. The overall networks, i.e. virtual limits between regions of the 317 same radius, seemed to be almost unchanged in the horizontal direction during proving while 318 bubbles underwent overall growth. From Fig. 7 and a complete set of maps of radii, dough 319 evolution seemed to be related to the starting structure of the dough. Image processing 320 methods on maps of radii would be useful for tracking purposes.

\section{Discussion}

In this section, we first discuss the simulation of $T_{2} \chi$ and the behavior of the signal. We 323 then explain the limitations of the method. Finally, results on non-yeasted dough and dough 324 during proving will be analyzed in the light of this previous discussion.

\subsection{Simulated evolution of $T_{2} \chi$}

Simulation results concerning $T_{2} \chi$ as a function of bubble radius (Fig. 2) were original

327 and to our knowledge have never been reported in the literature. Several studies have been 328 undertaken to study NMR signal dephasing due to the presence of mesoscopic field 329 inhomogeneities in the static dephasing regime $[25,26]$. The relaxation constant was thus 330 found to be independent of cylinder or bubble radii. From the free induction decay due to 331 randomly distributed spherical particles, Yablonskiy [33] derived an expression for the 332 relaxation rate $R_{2}{ }^{\#}$ defined in section 2.1: 


$$
\mathrm{R}_{2}^{\#}=\frac{4 \pi}{9 \sqrt{3}} \varepsilon \cdot \gamma \cdot \Delta_{\chi} \cdot B_{0}
$$

Eq. (9) explains $T_{2} \chi$ shortening with increasing porosity (Fig. 3.a) or main magnetic field (Fig. 2.b), and the values are compatible with our present study. Indeed, if $\varepsilon=12.6 \%$, Eq. (9) resulted in $T_{2}{ }^{*}=1 /\left(R_{2}+R_{2}{ }^{*}\right)=12.2 \mathrm{~ms}$ and $T_{2} \chi=13 \mathrm{~ms}$ in our simulation.

We demonstrated three levels for $T_{2} \chi$ observation. First, the plateau in Fig. 2.a indicated that, for a given porosity (or density), $T_{2} \chi$ values are independent of bubble radii. This confirmed all the results encountered in mesoscopic scale studies. We showed that $T_{2} \chi$ was dependent on radius in a certain range (macroscopic scale) and thus constitutes a sensitive 341 indicator of the medium alveolar structure. The second plateau occurred for large bubbles 342 compared to voxel size. $T_{2} \chi$ was higher for larger bubbles because the signal from an air voxel 343 could not decrease with $T E$ due to the almost total absence of signal. In fact, heterogeneity 344 was high in the ROI corresponding to the second plateau with complete air voxels, whereas 345 for the first plateau each voxel contained small air bubbles. There were therefore more 346 susceptibility interfaces for small bubbles, thus explaining the lower $T_{2} \chi$ values. At a constant 347 radius, an increase in porosity means a reduction in the center to center distance ( $c c d$ ) and 348 thus intravoxel dephasing is greater, as described in [27] and in Eq. (1), explaining the 349 decrease of $T_{2} \chi$ with porosity.

Simulations at $1.5 \mathrm{~T}$ (Fig. 2.b) showed the potential of this method since there was a 351 greater difference between $T_{2} \chi_{\text {init }}$ values for low porosities and an augmentation of the $K$ 352 factor. By using Eq. (8) to reveal bubbles, the estimated radius dynamic is thus enlarged and 353 the algorithm would be more stable. Furthermore, a major gain in signal at high field would 354 make better resolution possible. This is valuable for the study of the very small bubbles 355 present in non-yeasted dough. Indeed, Bellido et al. reported a mean value of $100 \mu \mathrm{m}$ in wheat 356 flour dough [16]. 


\subsection{Limitations of the method}

Fig 2.a illustrates some drawbacks of the method. $T_{2} \chi$ is the same for small radii constituting the first plateau. Bubbles within this range cannot be discriminated. Fortunately, the process of dough proving with the growth of bubbles and the increase in local porosity was suited to this sensitive domain. Due to the shape of the $T_{2} \chi$ curve (Fig. 2.a), computation of $r(i, j)$ according to Eq. (8) worked well in the dynamic area between the two plateaux.

Although bread dough structure is highly heterogeneous (gaseous phase and dough films included), the model was quite accurate in terms of signal loss. A more accurate virtual description of the object will undoubtedly make possible the calculation of values of greater accuracy. In fact, the expansion of bubbles rapidly becomes heterogeneous and anisotropic (non-spherical and distorted bubbles) due to mechanisms of coalescence [23]. However, using the estimation algorithm, it is possible to distinguish between bubbles according to their radius. At this point, $r(i, j)$ values have to be considered as variables related to length rather than a physical variable. Babin et al. [22] found dough structures were heterogeneous and dependent on the recipe, with bubble radii in the range $0.05-0.3 \mathrm{~mm}$ at the very beginning of dough proving. Maps of the radii in the present study were thus thought to be overestimated.

\subsection{Non-yeasted dough and dough during proving}

There is a real value in combining SE and GE sequences to assess local porosity and signal loss and thus deduce bubble radii. A porosity map (Fig. 4) provides important information (density is a criterion used by bakers to distinguish between bread recipes or to evaluate defects originating from the flour or the different stages in bread making) but a map of radii (Fig. 5) supplements the scientific understanding of evolution of the dough structure, from small to large bubbles. Additionally, the field of view available with MRI offers the possibility of assessing macroscopic heterogeneities (scale of a few millimeters to centimeters) in samples of realistic size comparable with industrial practices. Several image 
processing techniques based on this map could be developed to classify or segment bubbles.

383 The histogram presented in Fig. 6 shows a Gaussian distribution of bubbles. Bellido et al. [16] 384 used microcomputed tomography on dough and found a log-normal distribution. These first 385 results were encouraging for the study of non-yeasted bread dough.

Bikard et al. [23] analyzed the foaming phase during proving using 3D simulation. They 387 characterized the influence of parameters such as dough viscosity, kinetics or initial number 388 of bubbles on the evolution of average bubble radius over time. Fig. 9 obtained from maps of 389 radii at different times of proving is in good agreement with their results while looking at curve shapes. From a quantitative point of view, it was hard to compare as values were highly dependent on the above parameters that we could not quantify in our experiments.

Using digital image analysis, Rouille et al. [34] showed that reduction in the number of small bubbles $(\varnothing<1 \mathrm{~mm})$ was proportionally balanced by an increase in the number of larger bubbles ( $1 \mathrm{~mm}<\varnothing<2 \mathrm{~mm}$ ) during proving. This is also obvious from Figs. 7-8.

Image analysis-based methods $[17,20]$ showed limitations due to small sample size (magnetic resonance microscopy) and long imaging times. Even for small pixel size (115 $\mu \mathrm{m}$ ), resolution is not sufficient to detect small bubbles at the beginning of the proving time

398 [34]. Susceptibility-based methods are able to reveal information at a lower scale due to the expansion of field inhomogeneities and associated signal loss.

\section{Conclusion}

Because they arise from object-dependent field inhomogeneities, magnetic susceptibility

402 effects can be a source of quantitative data to characterize alveolar products such as bread 403 dough. While classic image analysis techniques are limited by image or temporal resolution, this new method combines local porosity, local signal loss and simulation predictions to assess bubble radii which can be smaller or the same order of magnitude than the voxel. 
407 different techniques or the use of a test object with known geometry and susceptibility. 408 Moreover, further simulation studies with more realistic object geometries to mimic bread 409 dough would make possible more quantitative results. Growth and distribution of bubbles 410 were observed during proving. Maps of radii gave information about dough structure and 411 evolution. The principle of the estimation algorithm was shown at $0.2 \mathrm{~T}$ and simulations 412 predicted greater distinction between bubble sizes at $1.5 \mathrm{~T}$, especially for low porosities 413 which are also encountered at key stages in breadmaking.

\section{Acknowledgments}

416 Council of Brittany for financial support. 


\section{References}

418 [1] Ludeke KM, Roschmann P and Tischler R. Susceptibility artefacts in NMR imaging. 419 Magn Reson Imaging 1985;3:329-343.

420 [2] Bakker CJG, Bhagwandien R, Moerland MA and Ramos LMP. Simulation of susceptibility artifacts in 2D and 3D Fourier transform spin-echo and gradient-echo magnetic resonance imaging. Magn Reson Imaging 1994;12:767-774.

[3] Fernandez-Seara MA and Wehrli FW. Postprocessing technique to correct for background gradients in image-based R2* measurements. Magn Reson Med 2000;44:358-366.

[4] Ogawa S, Menon RS, Tank DW, Kim SG, Merkle H, Ellermann JM and Ugurbil K. Functional brain mapping by blood oxygenation level-dependent contrast magnetic resonance imaging. A comparison of signal characteristics with a biophysical model. Biophys J 1993;64:803-812.

[5] Haacke EM, Xu YB, Cheng YCN and Reichenbach JR. Susceptibility weighted imaging (SWI). Magn Reson Med 2004;52:612-618.

[6] Sehgal V, Delproposto Z, Haacke EM, Xu Y, Neelavalli J, Haddar D, Tong KA, Wycliffe N, Kido DK and Reichenbach JR. Clinical applications of neuroimaging with susceptibilityweighted imaging. J Magn Reson Imaging 2005;22:439-450.

[7] Cunningham CH, Arai T, Yang PC, McConnell MV, Pauly JM and Conolly SM. Positive contrast magnetic resonance imaging of cells labeled with magnetic nanoparticles. Magn Reson Med 2005;53:999-1005.

[8] Bos C, Delmas Y, Desmouliere A, Solanilla A, Hauger O, Grosset C, Dubus I, Ivanovic Z, Rosenbaum J, Charbord $\mathrm{P}$ and others. In vivo MR imaging of intravascularly injected magnetically labeled mesenchymal stem cells in rat kidney and liver. Radiology 2004;233:781-789.

[9] Ittrich H, Lange C, Togel F, Zander AR, Dahnke H, Westenfelder C, Adam G and NolteErnsting C. In vivo magnetic resonance imaging of iron oxide-labeled, arterially-injected mesenchymal stem cells in kidneys of rats with acute ischemic kidney injury: Detection and monitoring at 3T. J Magn Reson Imaging 2007;25:1179-1191.

[10] Mowat P, Franconi F, Chapon C, Lemaire L, Dorat J, Hindre F, Benoit JP, Richomme P and Le Jeune JJ. Evaluating SPIO-labelled cell MR efficiency by three-dimensional quantitative T-2* MRI. NMR Biomed 2007;20:21-27.

[11] Beuf O, Briguet A, Lissac M and Davis R. Magnetic resonance imaging for the determination of magnetic susceptibility of materials. Journal of magnetic resonance. Series B 1996;112:111-118.

[12] Peeters JM, van Faassen EEH and Bakker CJG. Magnetic resonance imaging of microstructure transition in stainless steel. Magn Reson Imaging 2006;24:663-672.

[13] Robson P and Hall L. Identifying particles in industrial systems using MRI susceptibility artefacts. AIChE Journal 2005;51:1633-1640.

[14] Wong KK, Huang I, Kim YR, Tang H, Yang ES, Kwong KK and Wu EX. In vivo study of microbubbles as an MR susceptibility contrast agent. Magn Reson Med 2004;52:445-452.

[15] Niranjan K. An Introduction to Bubble Mechanics in Foods. In: Campbell GM, Webb C, Pandiella SS, Niranjan K, editors. Bubbles in Food. St Paul: Eagan Press, 1999. p. 3-9.

[16] Bellido GG, Scanlon MG, Page JH and Hallgrimsson B. The bubble size distribution in wheat flour dough. Food Res Int 2006;39:1058-1066.

[17] Bonny J-M, Rouille J, Della Valle G, Devaux M-F, Douliez J-P and Renou J-P. Dynamic magnetic resonance microscopy of flour dough fermentation. Magn Reson Imaging 2004;22:395-401. 
[18] Grenier A, Lucas T, Collewet G and Le Bail A. Assessment by MRI of local porosity in dough during proving. Theoretical considerations and experimental validation using a spinecho sequence. Magn Reson Imaging 2003;21:1071-1086. [19] Ishida N, Takano H, Naito S, Isobe S, Uemura K, Haishi T, Kosse K, Koizumi M and Kano H. Architecture of baked breads depicted by a magnetic resonance imaging. Magn Reson Imaging 2001;19:867-874. [20] van Duynhoven JPM, van Kempen GMP, van Sluis R, Rieger B, Weegels P, van Vliet LJ and Nicolay K. Quantitative assessment of gas cell development during the proofing of dough by magnetic resonance imaging and image analysis. Cereal Chem 2003;80:390-395. [21] Wagner MJ, Loubat M, Sommier A, Le Ray D, Collewet G, Broyart B, Quintard H, Davenel A, Trystram G and Lucas T. MRI study of bread baking: Experimental device and MRI signal analysis. International Journal of Food Science and Technology 2008;43:11291139.

[22] Babin P, Della Valle G, Chiron H, Cloetens P, Hoszowska J, Pernot P, Re?guerre AL, Salvo L and Dendievel R. Fast X-ray tomography analysis of bubble growth and foam setting during breadmaking. Journal of Cereal Science 2006;43:393-397.

480

481

482

483

484

485

486

487

488

489 [23] Bikard J, Coupez T, Della Valle G and Vergnes B. Simulation of bread making process using a direct 3D numerical method at microscale: Analysis of foaming phase during proofing. J Food Eng 2008;85:259-267.

[24] Babin P, Della Valle G, Dendievel R, Lourdin D and Salvo L. X-ray tomography study of the cellular structure of extruded starches and its relations with expansion phenomenon and foam mechanical properties. Carbohydrate Polymers 2007;68:329-340.

[25] Yung K-T. Empirical models of transverse relaxation for spherical magnetic perturbers. Magn Reson Imaging 2003;21:451-463.

[26] Sukstanskii AL and Yablonskiy DA. Theory of FID NMR signal dephasing induced by mesoscopic magnetic field inhomogeneities in biological systems. J Magn Reson 2001;151:107-117.

[27] De Guio F, Benoit-Cattin H and Davenel A. Signal decay due to susceptibility-induced intravoxel dephasing on multiple air-filled cylinders: MRI simulations and experiments. Magn Reson Mater Phy 2008;21:In press.

[28] Benoit-Cattin H, Collewet G, Belaroussi B, Saint-Jalmes H and Odet C. The SIMRI project: a versatile and interactive MRI simulator. J Magn Reson 2005;173:97-115.

[29] Ruan RR, Wang XA, Chen PL, Fulcher RG, Pesheck P and Chakrabarti S. Study of water in dough using nuclear magnetic resonance. Cereal Chem 1999;76:231-235.

[30] Grenier A, Lucas T, Davenel A, Cambert M, Le Bail A and Mariette F. Assessment of NMR relaxation times and ice fraction in frozen dough. J Agr Food Chem 2008:submitted.

[31] Lodi A, Abduljalil AM and Vodovotz Y. Characterization of water distribution in bread during storage using magnetic resonance imaging. Magn Reson Imaging 2007;25:1449-1458.

[32] Cauvain SP, Whitworth MB and Alava JM. The Evolution of Bubble Structure in Bread Doughs and Its Effect on Bread Structure. In: Campbell GM, Webb C, Pandiella SS, Niranjan K, editors. Bubbles in Food. St Paul: Eagan Press, 1999. p. 85-88.

[33] Yablonskiy DA. Quantitation of intrinsic magnetic susceptibility-related effects in a tissue matrix. Phantom study. Magn Reson Med 1998;39:417-428.

[34] Rouillé J, Della Valle G, Devaux MF, Bonny J-M and Renou JP. Effect of flour minor components on bubble growth in bread dough during proofing assessed by magnetic resonance imaging. J Agr Food Chem 2005;53:3986-3994. 
512 Figure 1: Overview of simulation framework. Field inhomogeneities map was dependent on 513 object parameters $(r, c c d, \Delta \chi, N b)$ and was an input of MRI simulator SIMRI. $T_{2} \chi$ was 514 determined for each object from ROI in GE simulated images at different TE from 4 to $12 \mathrm{~ms}$.

515 Figure 2: $T_{2} \chi$ value as a function of bubble radii at different porosities with sigmoid fitting curves. a) At $B_{0}=0.2 \mathrm{~T}$. b) At $B_{0}=1.5 \mathrm{~T}$. Three stages were identified: an initial plateau where $T_{2} \chi$ was only dependent on porosity (density), a rapid increase in $T_{2} \chi$ value and a

518 second plateau corresponding to high air proportion in voxels.

519 Figure 3: a) $K$ and $T_{2} \chi_{\text {init }}$ as functions of porosity. b) $r_{\text {break }}$ and $\lambda$ as functions of porosity. 520 Second order polynomial relationship with porosity was found for each simulation parameter.

521 Figure 4: Porosity map of non-yeasted bread dough. Large bubbles can be distinguished as 522 high-value pixels. The squared reference ROI is represented.

523 Figure 5: Map of estimated radii of bubbles in non-yeasted bread dough. Contrast 524 enhancement between the different regions of bubble sizes highlighting dough structure with 525 small bubbles surrounded by larger bubbles, etc.

526 Figure 6: Histogram with Gaussian distribution $\left(\sigma=0.062, \mu=0.37, \mathrm{R}^{2}=0.991\right)$ of the non527 yeasted dough computed on the reference ROI with a 0.02 pix $_{\text {ima }}$ interval.

528 Figure 7: Map of estimated radii of bubbles in bread dough during proving. a) At $t=7 \min \mathbf{b}$ )

529 At $\mathrm{t}=12.5 \mathrm{~min} \mathbf{c})$ At $\mathrm{t}=18 \mathrm{~min}$ showing distribution and growth of bubbles.

530 Figure 8: Histograms extracted from maps of radii showing distribution of bubble sizes at 531 three proving times.

532 Figure 9: Average estimated radius as a function of proving time. 


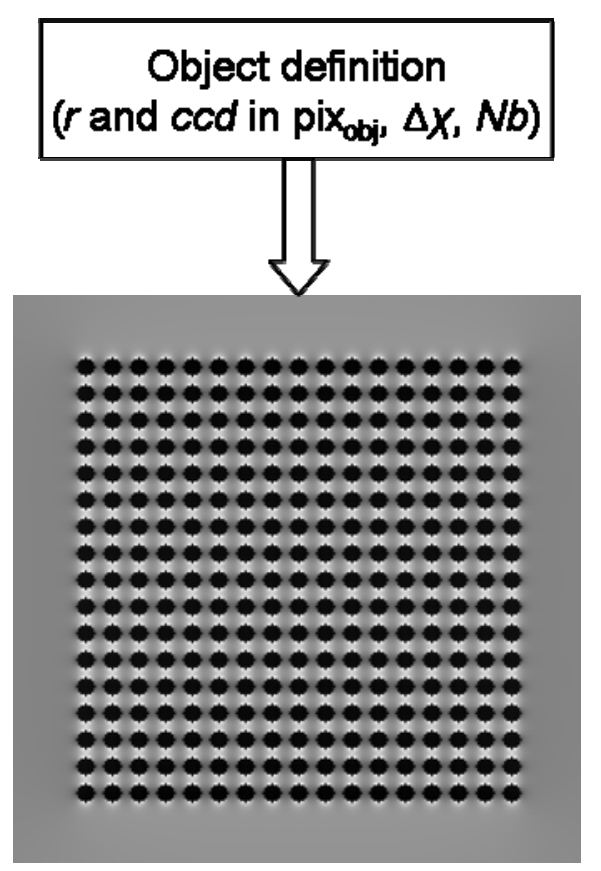

Field inhomogeneities $\left(\Delta B / B_{0}\right)$

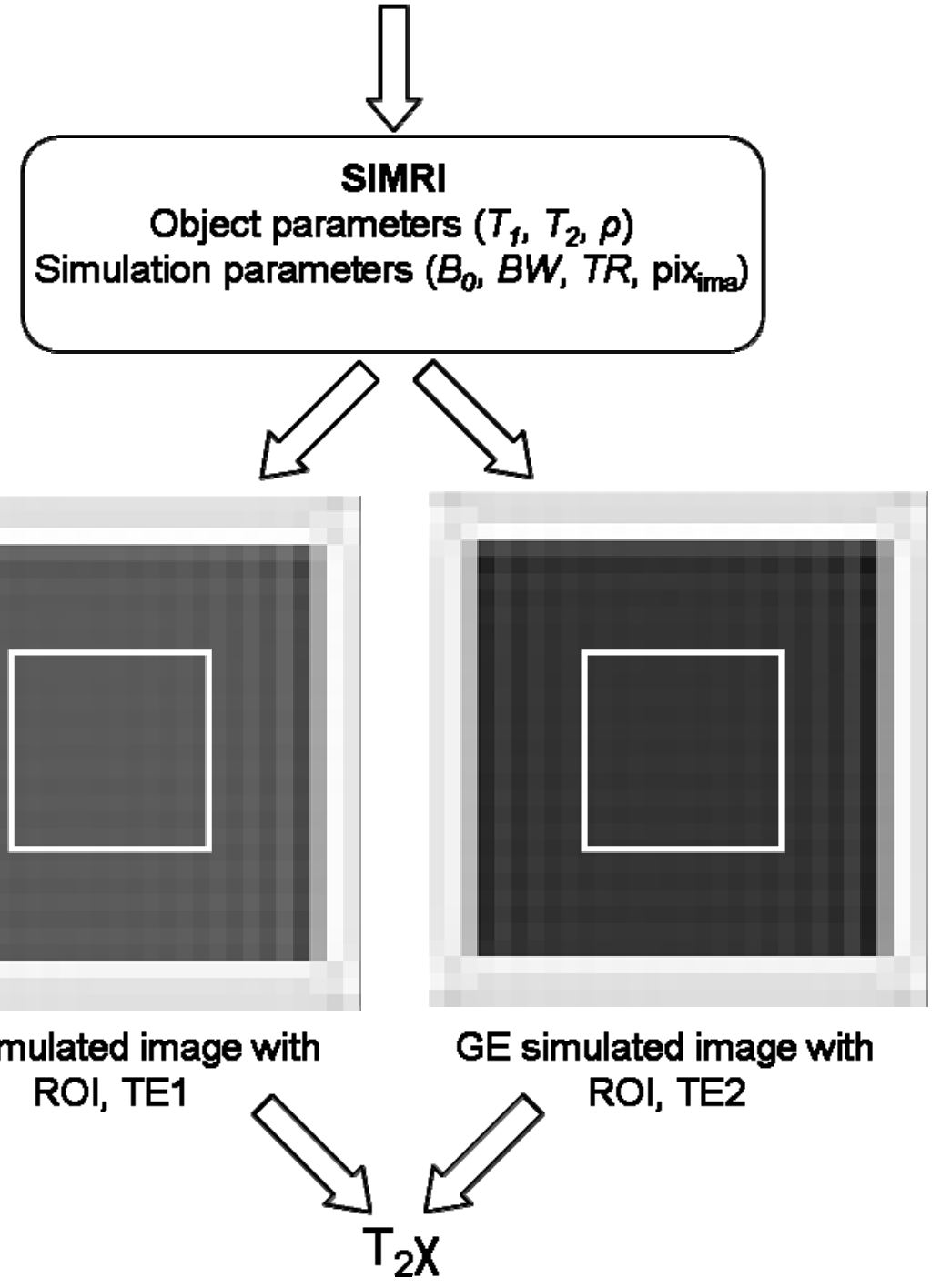

Fig. 1 
$T_{2 X}$ function of bubbles radius at $0.2 \mathrm{~T}$

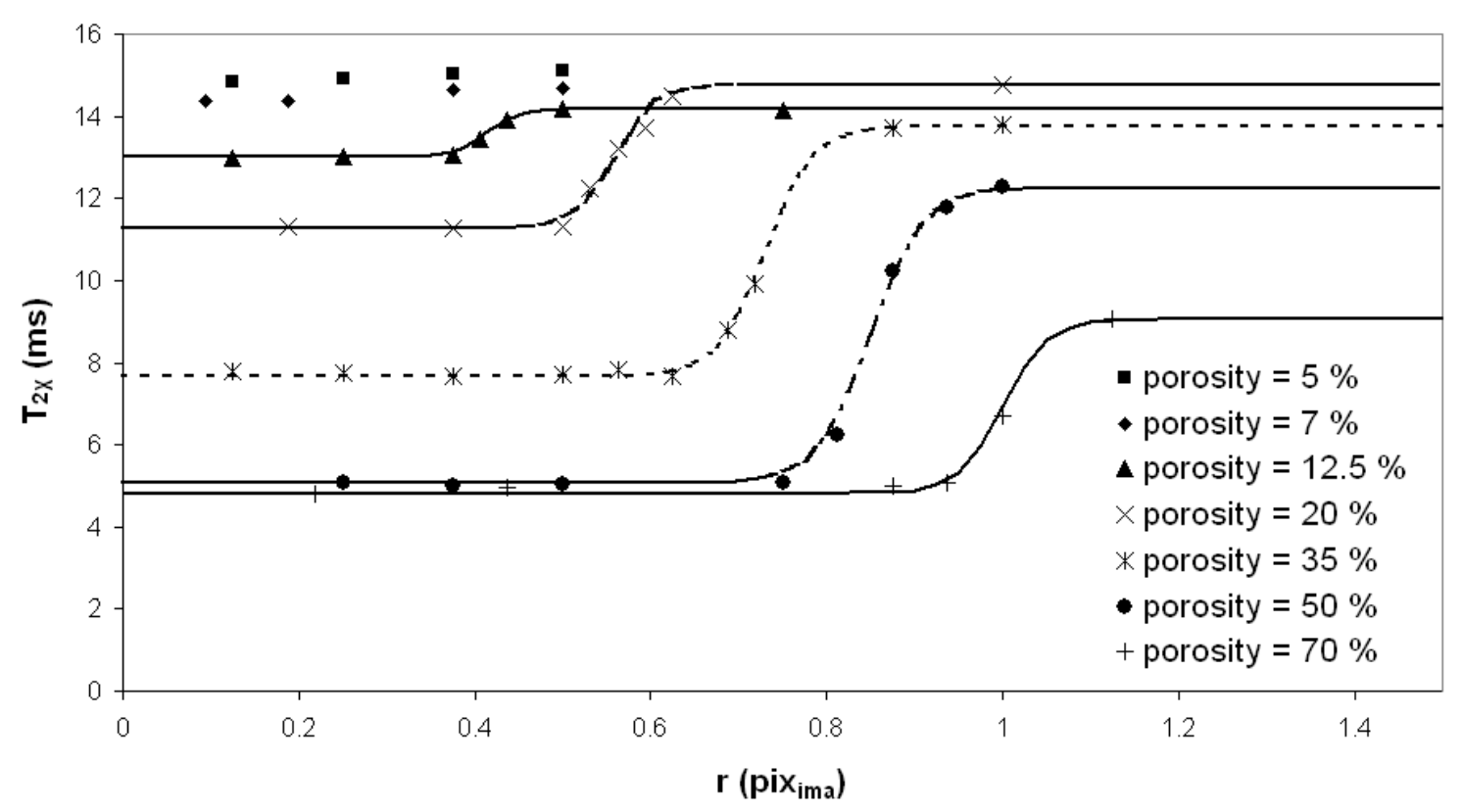

535

$536 \quad$ Fig2a

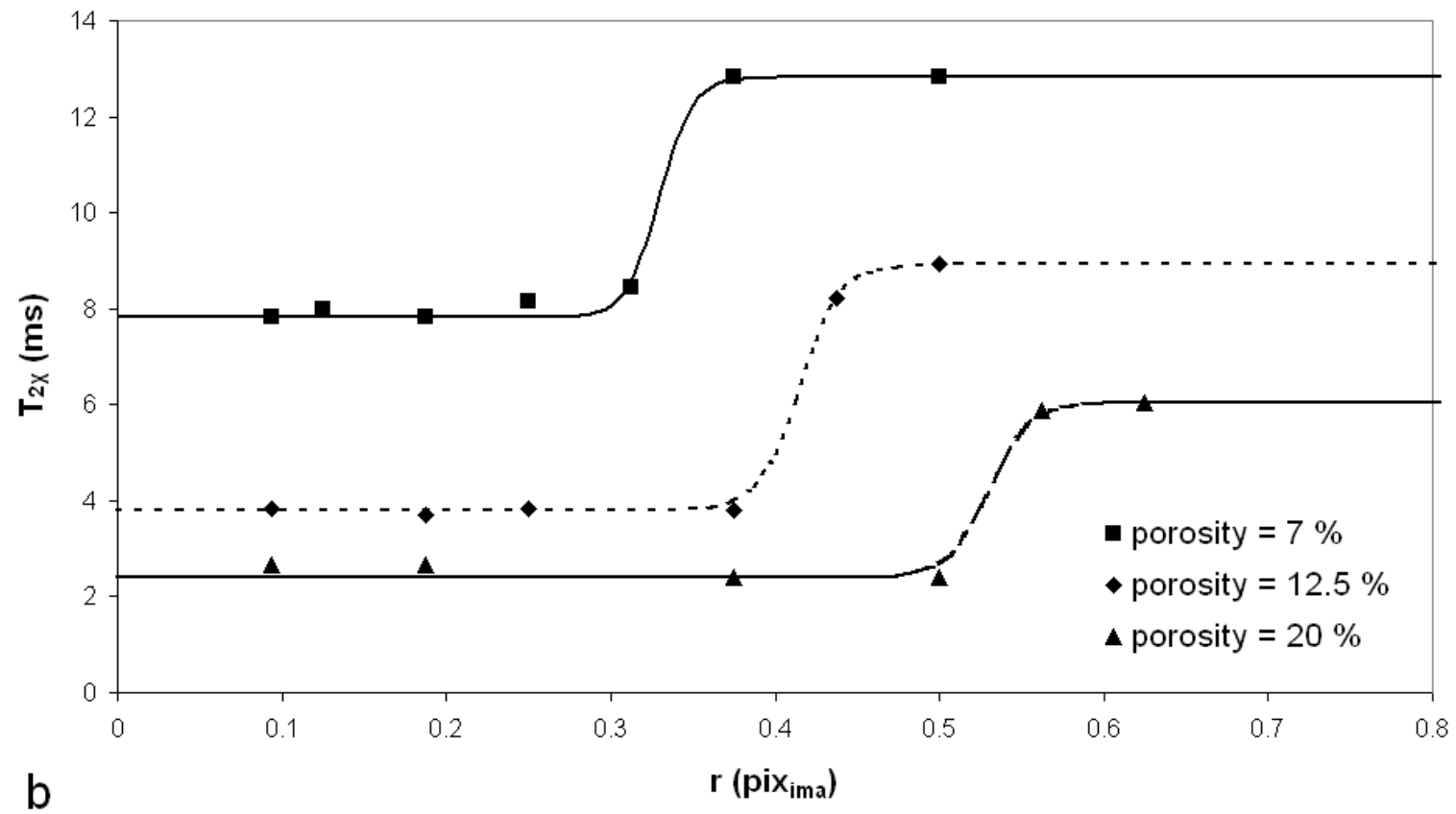

$538 \quad$ Fig. $2 b$ 


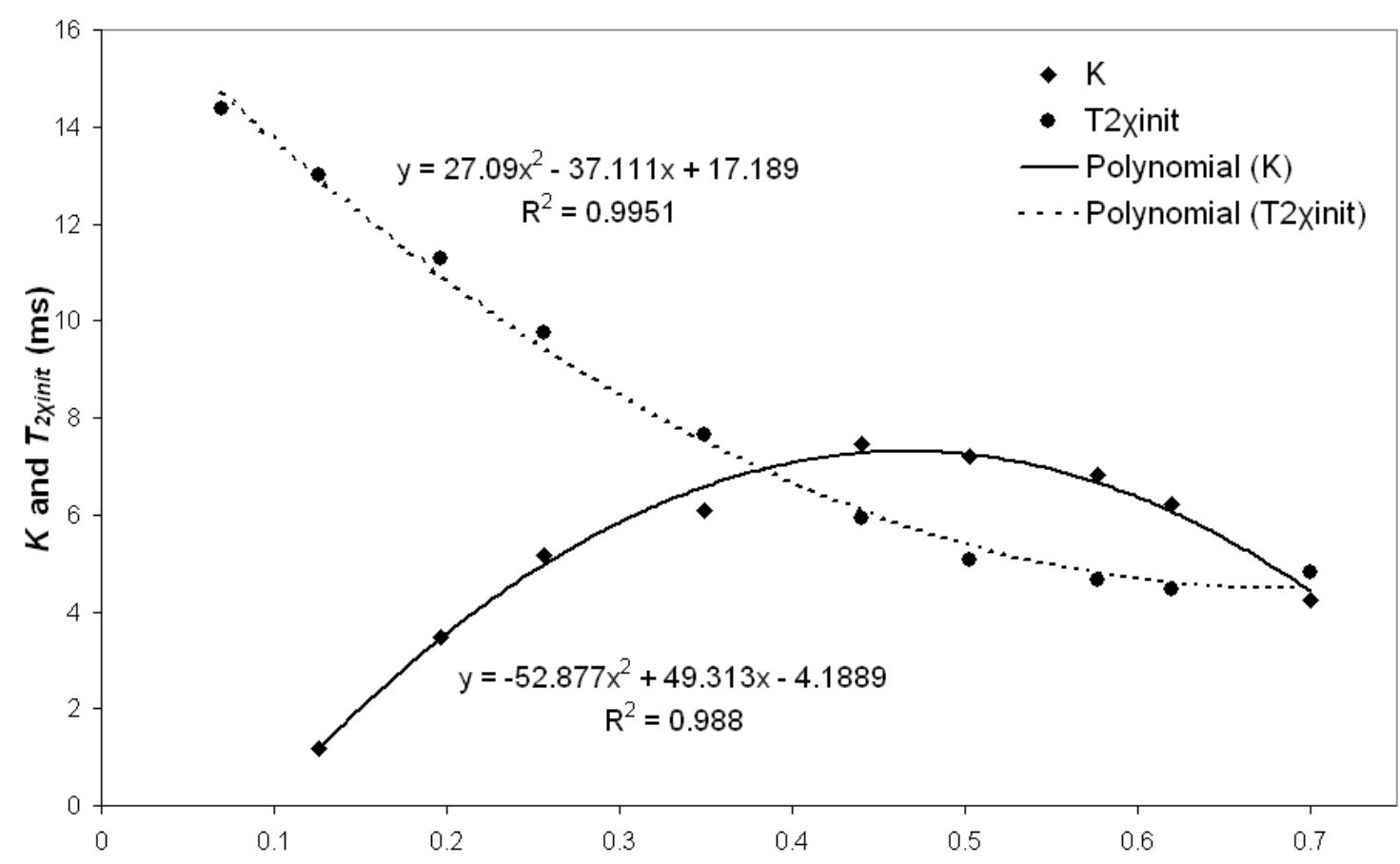

539

a

Porosity (\%)

$540 \quad$ Fig.3a

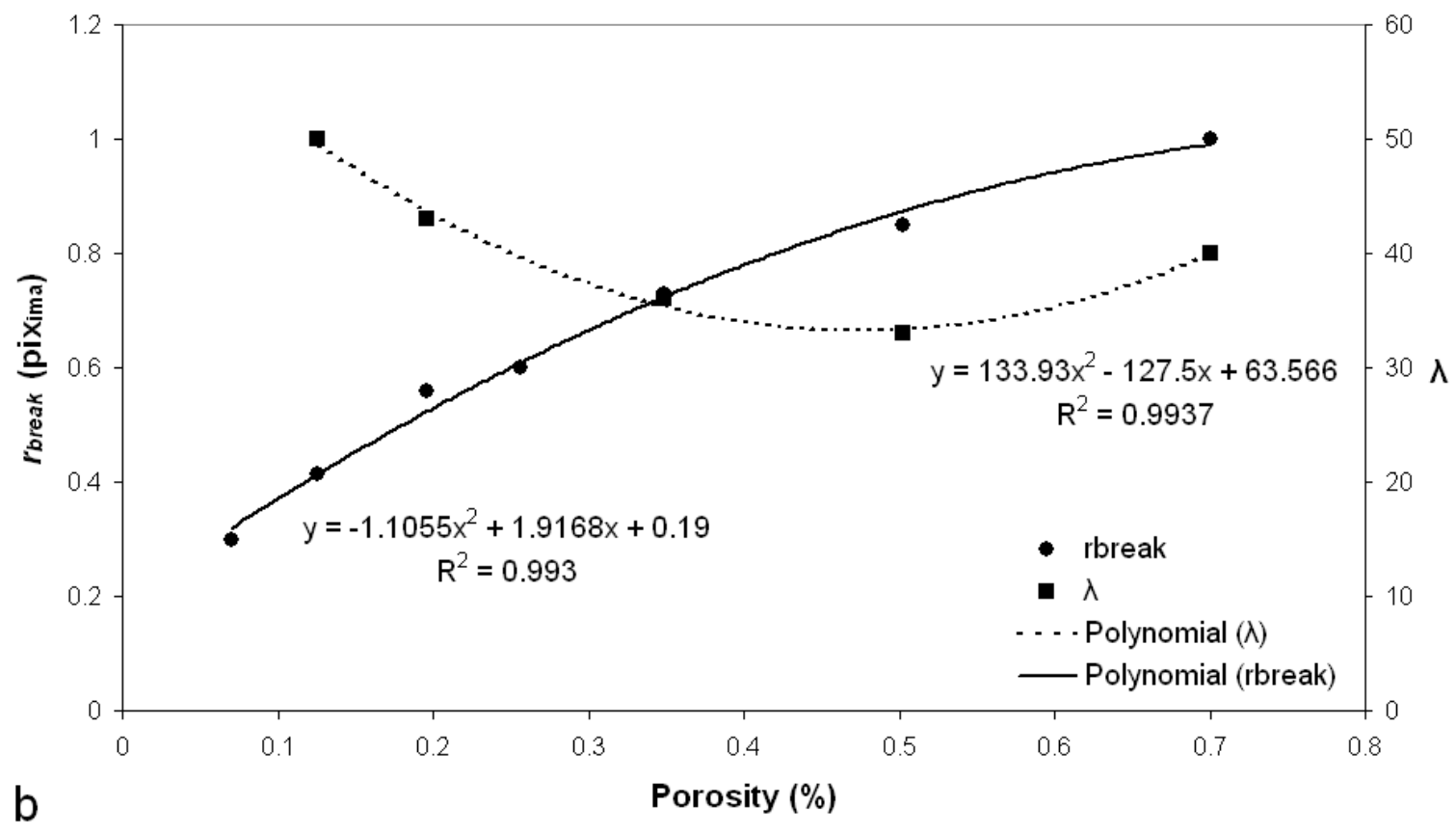

541

Fig.3b 


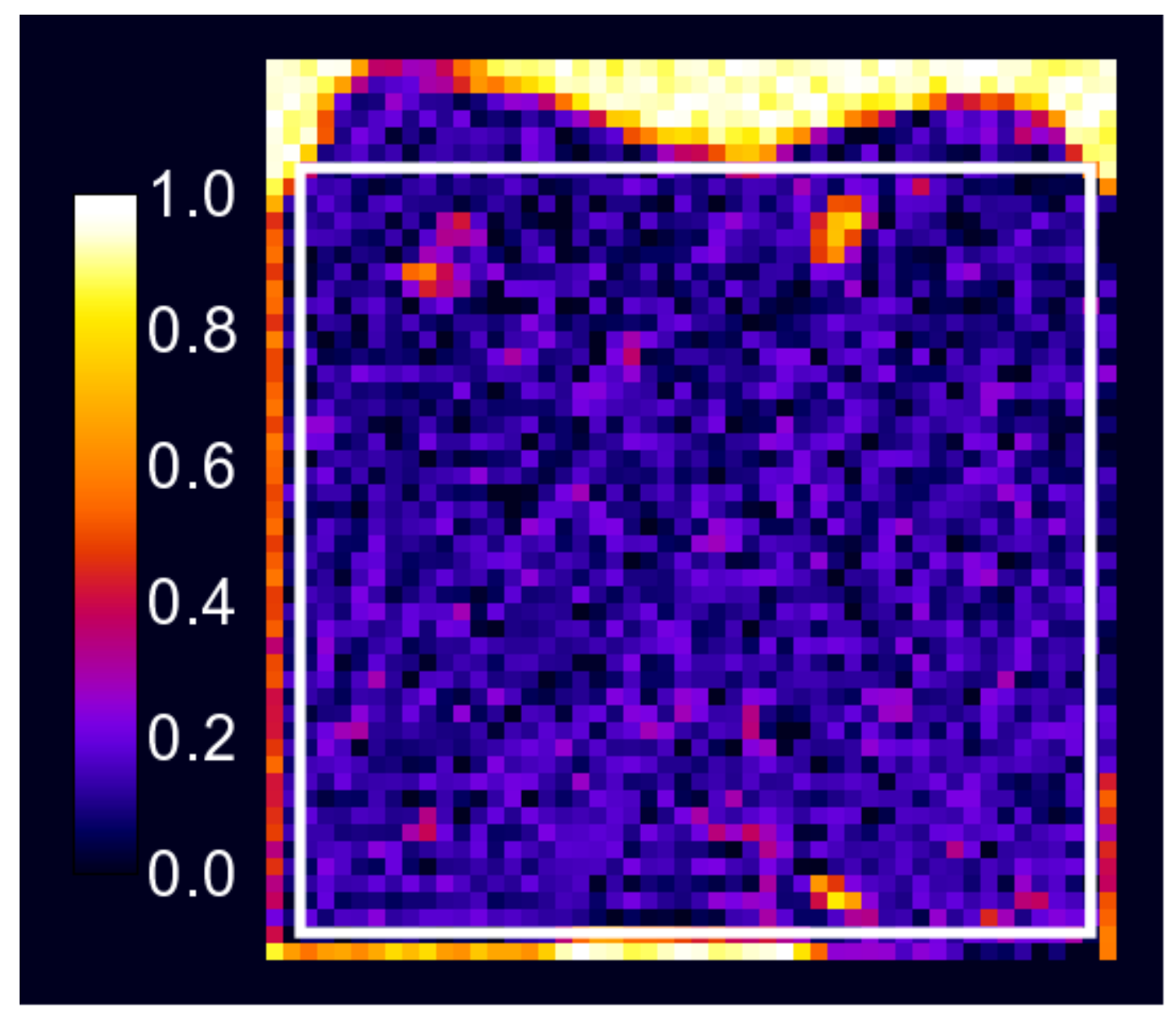

543

Fig. 4 


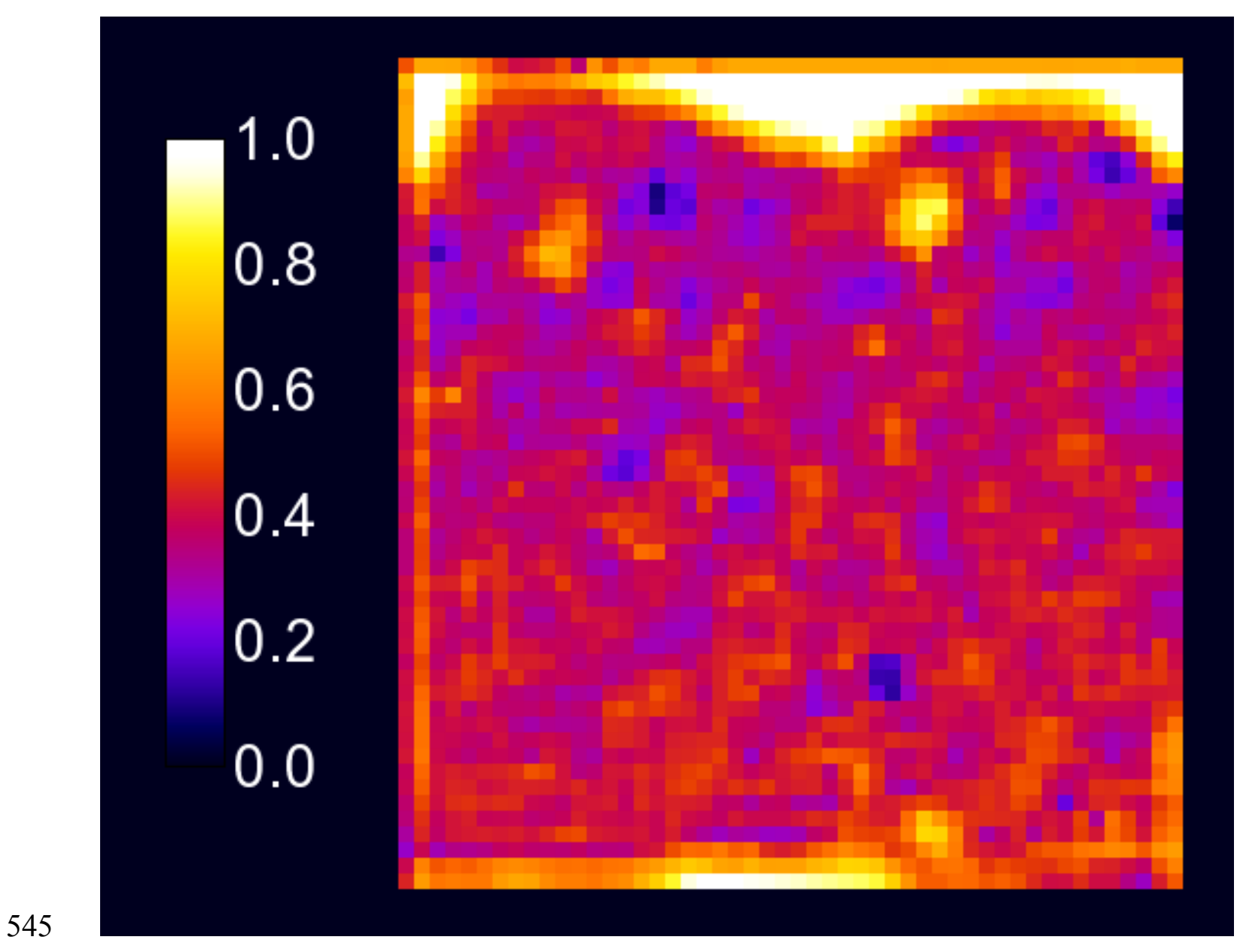

$546 \quad$ Fig. 5 


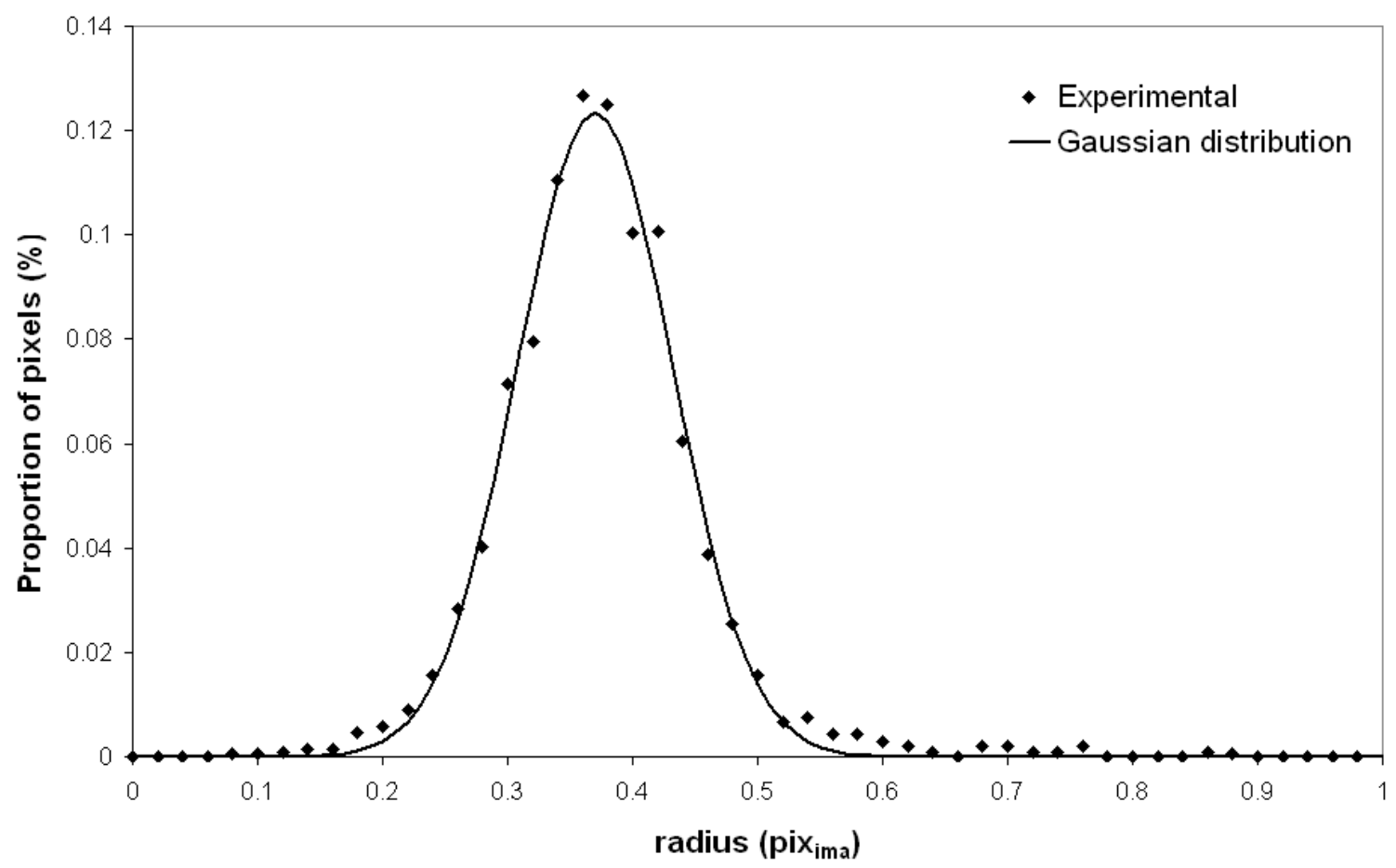

547

$548 \quad$ Fig.6 


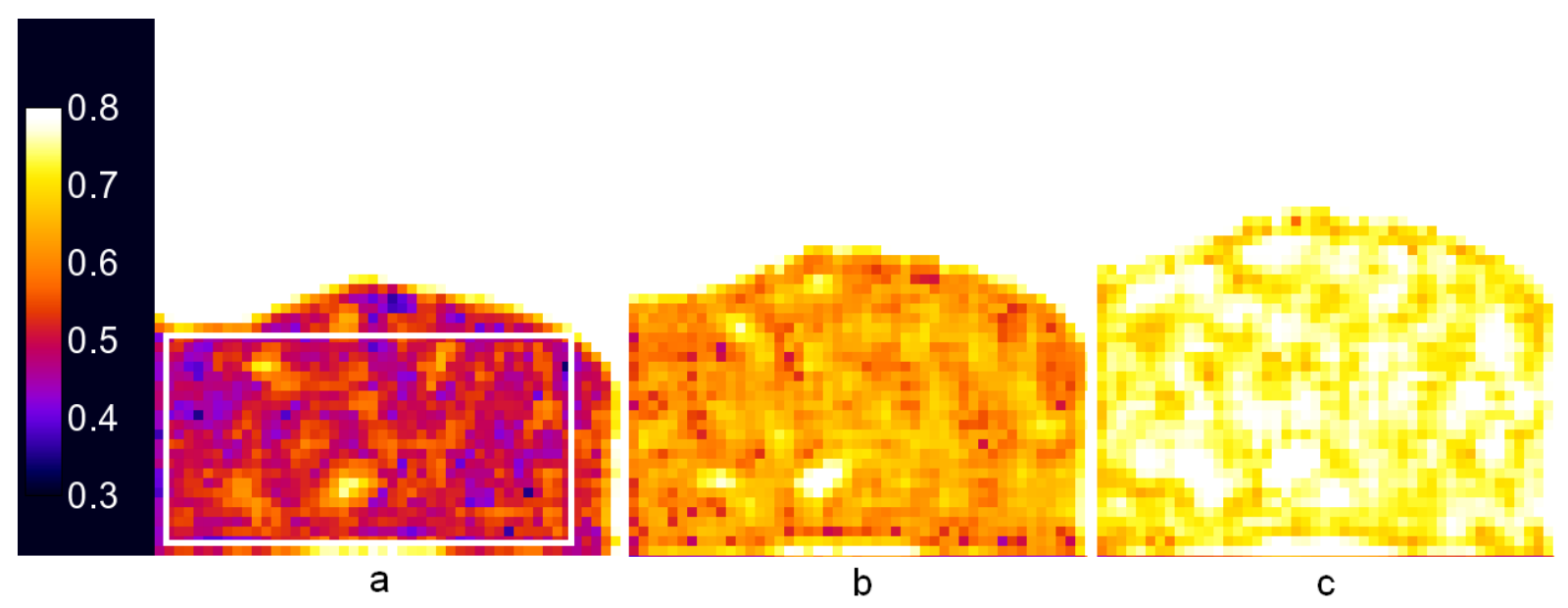

$550 \quad$ Fig7 


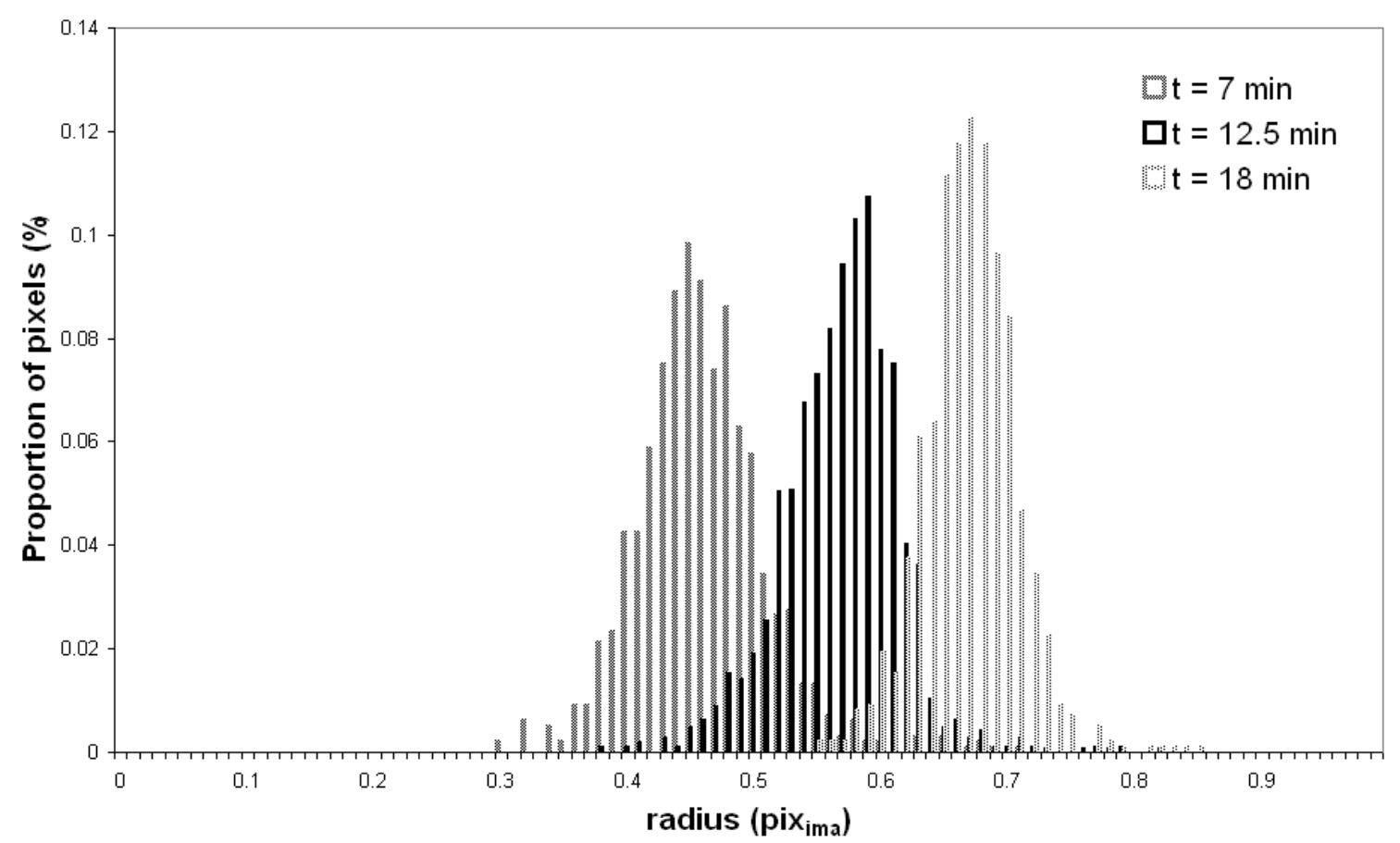

551

$552 \quad$ Fig.8 


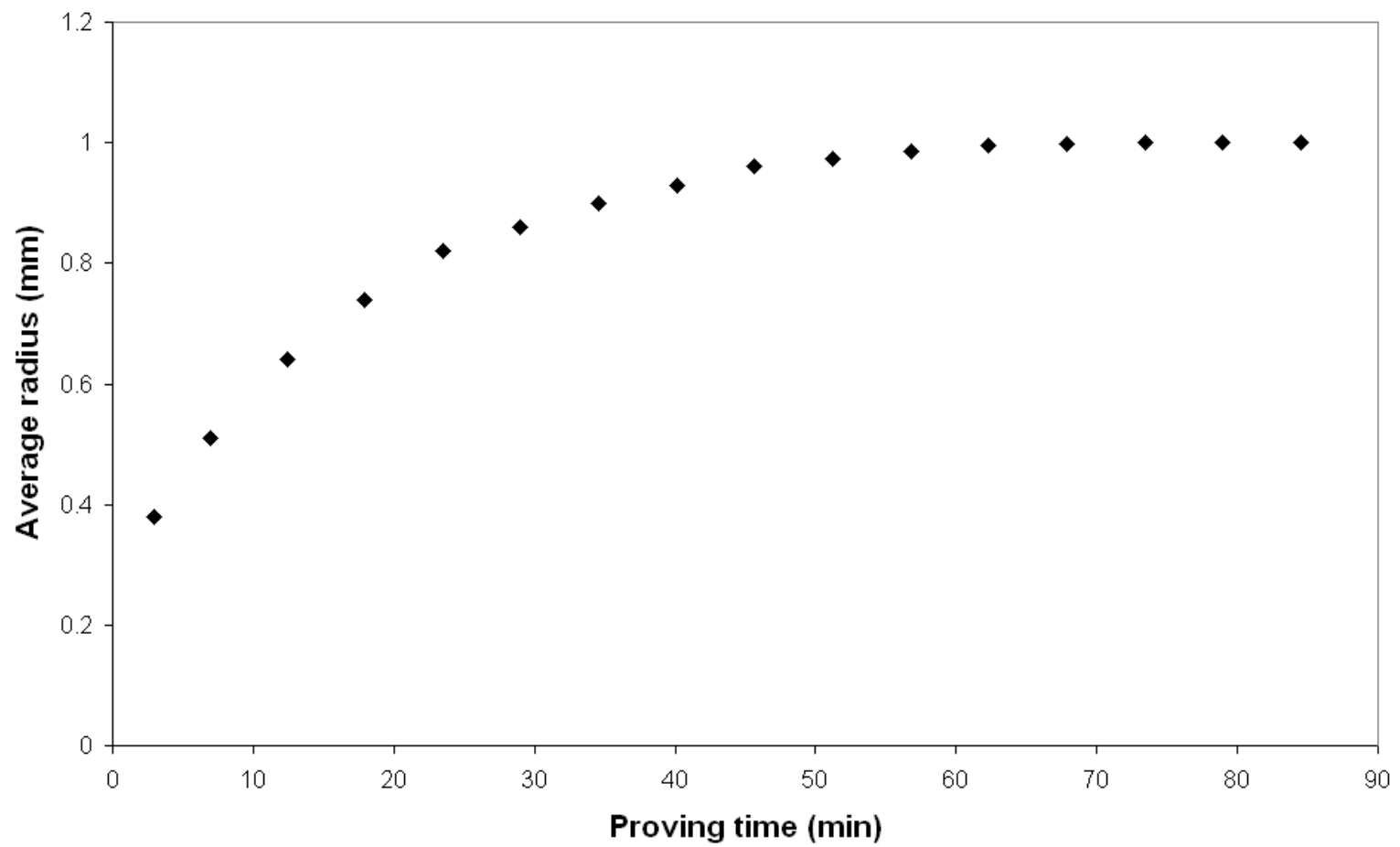

553

554 Fig.9

555 